\title{
Constitutive Expression of Truncated MYC2 Affects Expression of Jasmonate-Responsive Genes
}

\author{
Sun Hyun Chang ${ }^{1}$, Taeyoung Um ${ }^{1}$, Sangyool Lee ${ }^{1}$, Geupil Jang ${ }^{1}$, Ju-Kon Kim ${ }^{2}$, Yang Do Choi ${ }^{1}$ \\ ${ }^{1}$ Department of Agricultural Biotechnology and Research Institute of Agriculture and Life sciences \\ Seoul National University \\ Seoul 08826, Korea \\ dacadaca@snu.ac.kr; tyum@snu.ac.kr; leesangyool@snu.ac.kr; yk3@snu.ac.kr; choiyngd@snu.ac.kr \\ ${ }^{2}$ Graduate School of International Agricultural Technology and Crop Biotechnology Institute \\ Green BioScience and Technology, Seoul National University \\ Pyeongchang 25354, Korea \\ jukon@snu.ac.kr
}

\section{Extended Abstract}

After land plants started to establish the first continental vegetation 4.5 million years ago, they have evolved various defense mechanisms against environmental stresses to survive in terrestrial habitats. In plant biology defense mechanisms have been extensively studied to develop the transgenic crops with stress-resistance traits because environmental stresses severely reduce plant development and productivity. Jasmonic acid (JA) is an important phytohormone that mediates plant responses to abiotic stresses such as drought and cold. Recent advances in molecular biology suggested that regulation of transcription networks is the most crucial step in hormone responses. In JA response, MYC2 transcription factor governs transcriptional expression of JA-responsive genes as a key regulator. However, it was shown that overexpression of fulllength of $M Y C 2$ cDNA has little effects on JA response, and previous many of studies suggested that interaction between MYC2 and JAZs, repressor proteins for JA response, is deeply involved in this phenomenon. MYC2, a basic helix-loophelix transcription factor, contains JAZ-interacting domain in its $\mathrm{N}$-terminal region upstream of the basic helix-loop-helix domain. In normal or JA-free conditions, MYC2 exists as a complex with JAZs and has no activity to promote expression of JA-responsive genes. However, in stress or JA-treated conditions, the JAZs are degraded by E3 ubiquitin ligasemediated proteolysis pathway, and MYC2 can function as a transcription factor. In this study, we tried to generate modified MYC2 (MYC2 $\triangle \mathrm{N})$ without activity to interact with JAZs, and analysed the effect of MYC2 $\Delta \mathrm{N}$ on endogenous JA response in plants. In the MYC $2 \Delta \mathrm{N}$, the JAZ-interacting domain was deleted. To analyse the interaction between MYC2 $\triangle \mathrm{N}$ and JAZs, yeast two-hybrid assay was performed. From this approach, we found that MYC2 $\Delta \mathrm{N}$ did not interact with intact MYC2-binding JAZs such as JAZ2, 3, 5, 8 and 9, indicating that the JAZ-interacting activity is abolished in the truncated MYC2. To understand the effects of MYC2 $\triangle \mathrm{N}$ overexpression on endogenous JA response, we generated the transgenic plants transformed with $35 S:: M Y C 2$ and $35 S:: M Y C 2 \triangle N$, respectively, and investigated JA response by analysing expression levels of JA-responsive genes in these transgenic plants. In $35 S: \because M Y C 2 \triangle N$ plants, expression levels of JA-responsive genes such as $A O S$ and JAZ9 were higher than that in wild type. However expression levels of these genes were almost identical between $35 S:: M Y C 2$ and wild-type plants, indicating that JA response is enhanced in the $35 S:: M Y C 2 \triangle N$ plants. When the JA responses were visualized using proJAZ9::erGFP, fluorescent signals were detected in the $M Y C 2 \triangle N$-overexpressing plants even in JA-untreated condition, whereas $35 S:: M Y C 2$ and wild type plants grown at the same condition did not show the signals. The root growth patterns further supported the enhanced JA response in $35 S:: M Y C 2 \triangle N$ transgenic plants. In JA-untreated condition, the root length of $35 S: \because M Y C 2$ plants were also almost identical to that of wild type, whereas $35 S:: M Y C 2 \triangle N$ plants developed significantly shorter roots as the wild type plants grown in JA-treated condition. Collectively, these findings indicate that endogenous JA response can be enhanced by the expression of $M Y C 2 \triangle N$, proposing a potential tool to study stress response mechanism of plants. 